Published in final edited form as:

Phytomedicine. 2019 April ; 57: 49-56. doi:10.1016/j.phymed.2018.12.002.

\title{
Artemisia annua and Artemisia afra tea infusions vs. artesunate- amodiaquine (ASAQ) in treating Plasmodium falciparum malaria in a large scale, double blind, randomized clinical trial
}

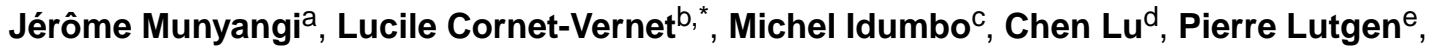 \\ Christian Perronne $^{f}$, Nadège Ngombe ${ }^{g}$, Jacques Bianga ${ }^{h}$, Bavon Mupenda ${ }^{i}$, Paul Lalukalaj, \\ Guy Mergeaik, Dieudonné Mumba', Melissa Towler ${ }^{\mathrm{m}}$, Pamela Weathers ${ }^{\mathrm{m}}$ \\ aFaculté de Médecine Université de Kolwezi-Lualaba, Congo DRC \\ bVice Présidente de La Maison de l'Artemisia(association Loi 1901), 20 rue Pierre Demours, \\ 75017 Paris, France \\ 'Centre de Santé de Lubile, Maniema, Congo DRC \\ dDepartment of Mathematics, Worcester Polytechnic Institute, USA \\ eAssociation IFVB-BELHERB, Luxembourg \\ fFaculté de Médecine de Paris IDF Ouest, France \\ gFaculté de Pharmacie, Université de Kinshasa, Congo DRC \\ hProgramme National Lutte Contre le Paludisme, Maniema, Congo DRC \\ 'Ecole de Santé Publique Université de Kinshasa, Congo DRC \\ jMinistère Provincial de Santé Publique Maniema, Congo DRC \\ kUniversité de Liège, Belgium \\ 'Faculté de Médecine Université de Kinshasa, Congo DRC \\ mDepartment of Biology and Biotechnology, Worcester Polytechnic Institute, USA
}

\begin{abstract}
Background and objective: Prior small-scale clinical trials showed that Artemisia annua and Artemisia afra infusions, decoctions, capsules, or tablets were low cost, easy to use, and efficient in curing malaria infections. In a larger-scale trial in Kalima district, Democratic Republic of Congo, we aimed to show $A$. annua and/or $A$. afra infusions were superior or at least equivalent to artesunate-amodiaquine (ASAQ) against malaria.
\end{abstract}

\footnotetext{
*Corresponding author. 1cv@ maison-artemisia.org (L. Cornet-Vernet).

Conflict of interest

We confirm that there are no known conflicts of interest associated with this publication and there has been no significant financial support for this study that could have influenced its outcome.

Supplementary materials

Supplementary material associated with this article can be found, in the online version, at doi:10.1016/j.phymed.2018.12.002.
} 
Methods: A double blind, randomized clinical trial with 957 malaria-infected patients had two treatment arms: 472 patients for ASAQ and 471 for Artemisia (248 A. annua, 223 A. afra) remained at end of the trial. ASAQ-treated patients were treated per manufacturer posology, and Artemisia-treated patients received 1 1/d of dry leaf/twig infusions for $7 \mathrm{~d}$; both arms had $28 \mathrm{~d}$ follow-up. Parasitemia and gametocytes were measured microscopically with results statistically compared among arms for age and gender.

Results: Artemisinin content of $A$. afra was negligible, but therapeutic responses of patients were similar to A. annua-treated patients; trophozoites cleared after $24 \mathrm{~h}$, but took up to $14 \mathrm{~d}$ to clear in ASAQ-treated patients. D28 cure rates defined as absence of parasitemia were for pediatrics 82, 91, and 50\% for A. afra, A. annua and ASAQ; while for adults cure rates were 91, 100, and 30\%, respectively. Fever clearance took $48 \mathrm{~h}$ for ASAQ, but $24 \mathrm{~h}$ for Artemisia. From D14-28 no Artemisia-treated patients had microscopically detectable gametocytes, while 10 ASAQ-treated patients remained gametocyte carriers at D28. More females than males were gametocyte carriers in the ASAQ arm but were unaffected in the Artemisia arms. Hemoglobin remained constant at 11 $\mathrm{g} / \mathrm{dl}$ for $A$. afra after D1, while for $A$. annua and ASAQ it decreased to $9-9.5 \mathrm{~g} / \mathrm{dl}$. Only $5.0 \%$ of Artemisia-treated patients reported adverse effects, vs. $42.8 \%$ for ASAQ.

Conclusion: $A$. annua and $A$. afra infusions are polytherapies with better outcomes than ASAQ against malaria. In contrast to ASAQ, both Artemisias appeared to break the cycle of malaria by eliminating gametocytes. This study merits further investigation for possible inclusion of Artemisia tea infusions as an alternative for fighting and eradicating malaria.

\section{Keywords}

ACT; ASAQ; Artemisinin; Malaria; Clinical Trial; Tea infusion

\section{Background}

The Democratic Republic of Congo (DRC) has one of the highest malaria rates. In 2016 > 50,000 people died, mainly children (WHO, 2017 http://apps.who.int/iris/bitstream/ $10665 / 259492 / 1 / 9789241565523$-eng.pdf). Maniema province is a holo-endemic zone with $>80 \%$ of the population infected (Programme National de Lutte contre le Paludisme, 2015. Rapport Malaria. Kinshasa, RDC; PNLP). In 2005, Artemisinin Combination Therapy (ACT) became the first line treatment for all in the DRC.

Artemisia annua L. and Artemisia afra Jacq. ex Willd., of Chinese and South African origin, respectively, were used for centuries medicinally as infusions or powders to treat malaria (Willcox et al., 2004; Weathers et al., 2014; Liu et al., 2010). Both showed in vitro efficacy against Plasmodium falciparum (Kraft et al., 2003; Moyo et al., 2016; Gathirwa et al., 2007; Liu et al., 2010). For generations, $A$. afra was an integral part of the pharmacopeia in many regions of DRC and other areas of eastern and southern Africa (FAO Forestry paper 67: 6873, Rome. http://www.fao.org/docrep/015/an797e/an797e00.pdf). In recent case studies, administration of dried $A$. annua leaf tablets successfully cured 18 patients with severe malaria who did not respond to therapy with ACT (artemether-lumefantrine) and i.v.artesunate (Daddy et al., 2017). 
Medicinal plants represent the main, if not the only treatment accessible to much of humanity (WHO, 2014 http://www.who.int/medicines/publications/traditional/ trm_strategy14_23/en/) and may offer a low cost and potentially more widely available alternative to ACTs. A. annua is grown in commercial plantations and by small stake-holder farmers in at least 39 countries, 33 of which are in Africa (Supplemental Material). For traditional medicine, WHO provided guidelines (WHO, $2000 \mathrm{http} / / / \mathrm{apps}$.who.int/iris/ bitstream/10665/66783/1/WHO_EDM_TRM_2000.1.pdf) and encouraged studies and clinical trials on herbal medicines (WHO, 2014). Subsequently, we launched a large-scale clinical trial aimed at confirming encouraging results from smaller-scale trials in Africa and South America with $A$. annua and $A$. absinthium. In those small trials cure rates of $>95 \%$ were reported (Mueller et al., 2000; Gebeyaw et al., 2010; Chougouo et al., 2012; ZimeDiawara et al., 2015).

\section{Methods}

\section{Plant material, handling and phytochemical analysis}

Field-grown leaves and twigs of Artemisia annua L. and Artemisia afra Jacq. ex Willd. (Asteraceae) were collected in France (PAR), Senegal (SEN), Burundi (BUR), and Luxembourg (LUX) and processed as described in Munyangi et al. (2018) where voucher ids also are listed. Artemisinin was measured using gas chromatography mass spectroscopy (GCMS) instead of HPLC to avoid artemisinin false positives (Smith et al., 2010).

Extraction and assay methods for phytochemical compounds are detailed in Munyangi et al. (2018) where phytochemical contents of both Artemisia sp. are documented: $A$. annua had $1.34-1.70 \mathrm{mg}$ artemisinin/g dry weight; $A$. afra had $0.036 \mathrm{mg}$ artemisinin/g dry weight.

\section{Study design}

The study was done in 2015 in Kalima health district, Pangi zone, Maniema province, in five areas: Kakutya, Kinkungwa, Kamundala, Lubile, and Kakozwa (for location map see supplemental material of Munyangi et al., 2018). Patients with uncomplicated malaria were enrolled in the three arms of the trial (Fig. 1) October to December 2015. The research protocol was registered and approved by the Comité d' Ethique de l' Ecole de Santé Publique de Kinshasa (MIN.RST/SG/180/001/2016) acting for the DRC Ministry of Health. All adult patients provided written consent to participate in the trial; minor patients were signed by parents. The study followed guidelines of the Declaration of Helsinki and Tokyo for humans.

\section{Criteria for inclusion of patients}

Patients were recruited by passive case finding. Eligibility of patients was restricted to adults or children $\geq 5 \mathrm{y}$ with axillary temperature of $237.5^{\circ} \mathrm{C}$ and parasitemia of $2000-200,000$ trophozoites $/ \mu$ l. Patients with these symptoms were excluded: severe malaria, undernourishment, repetitive vomiting \pm diarrhea, other concomitant infectious disease, known allergy to ACTs, pregnancy or breast feeding, cardiac, hepatic, or renal deficiencies. We also excluded patients treated with antimalarial drugs within $7 \mathrm{~d}$ preceding the trial or with antibiotics that might have antimalarial effects. Patients were enrolled and distributed randomly into a treatment arm (Fig. 1). Demographics of the study group are in Table 1. 
Drugs were distributed in numbered opaque envelopes selected randomly by each patient.

Clinical symptoms of enrolled patients are shown in Table S1.

\section{Clinical and laboratory analyses}

According to WHO procedures, a rapid diagnostic test (RDT) confirmed malarial infection. Kalima health district is a sentinel surveil-lance zone for malaria, so SDBioline Malaria Ag P.f./Pan test (batch nr 05FK60) was used on D0 for all patients. Thick and thin blood smears were microscopically analyzed for trophozoites and gametocytes, respectively. Trophozoites were measured per $\mu \mathrm{l}$ of blood; gametocytes were noted as present or absent to determine number of carriers. In each trial area, slides were scrutinized by two independent laboratories. In case of dubious or conflicting results, a PNLP expert was consulted. A random $10 \%$ of slides collected at different stages of the trial were quality-control checked by a third party. Hemoglobin level, asexual parasites, and presence of gametocytes were measured on D0. Hemoglobin was measured spectrophotometrically at $540 \mathrm{~nm}$ using Drabkin's Reagent at the peripheral health centers. Hemoglobin was also measured at the reference laboratory via hemoglobin analyzer; to settle value discrepancies, the reference lab value was used. After testing, patients were administered their first drug dose and a capillary blood sample was collected, dried on Whatman grade 3 filter paper, and stored for eventual genotyping. Patient follow-up including thick and thin blood smears was at D 1, 2, 3, 4, 7, 14, 21, and 28 post trial enrollment. From D0-D7, patients were treated in hospital to insure therapeutic compliance. Alanine (ALAT) and aspartate (ASAT) liver transaminases also were measured using the method of Reitman and Frankel (1957).

\section{Drug administration}

Artesunate-amodiaquine (ASAQ Winthrop, Sanofi-Aventis) was administered as tablets 25 $\mathrm{mg} / 67.5 \mathrm{mg}, 50 \mathrm{mg} / 135 \mathrm{mg}$, and $100 \mathrm{mg} / 270 \mathrm{mg}$. Manufacturer posology was: $4 \mathrm{mg}$ of artesunate and $10 \mathrm{mg}$ of amodiaquine $/ \mathrm{kg}$, once daily for $3 \mathrm{~d}$, followed by ASAQ placebo tablets for the remaining $4 \mathrm{~d}$. In the Artemisia arm, patients drank 0.331 of $A$. annua or $A$. afra infusion every $8 \mathrm{~h}$ for $7 \mathrm{~d}$ Infusion preparation was: $5 \mathrm{~g}$ of dried leaves and twigs of $A$. annua or $A$. afra to 11 of boiling water, infuse for $10 \mathrm{~min}$, and filter through sterilized $1 \mathrm{~mm}$ mesh. Pediatrics and adults received the same amount of Artemisia tea infusion; there was no adjustment for body weight.

The trial was double blinded as detailed in Munyangi et al. (2018). Briefly, $A$. annua and $A$. afra arms received the infusion plus ASAQ placebo from D1 to 7. The ASAQ arm received ASAQ tablets on D1-3 followed by ASAQ placebo on D4-7; these same patients also received Artemisia placebo from D1-7. ASAQ placebo consisted of pill-shaped saccharose/ glucose tablets purchased in a pharmacy. To conceive a placebo for Artemisia infusion was more challenging. WHO suggested using the drug at very low dose, so $A$. annua and $A$. afra placebos contained $0.2 \mathrm{~g} / \mathrm{l}$ of plant material and patients drank placebo infusion of one Artemisia species or the other. All trial personnel were unaware of arm assignments.

All treatments were administered inside health facilities and under medical control. In case of vomiting $30 \mathrm{~min}$ post-administration, the same drug was re-administered. For vomiting 60 min post-administration, no new administration took place. In case of persistent vomiting, 
the patient was excluded from the trial and their infection separately treated. Adverse events were recorded and defined by clinical observations and patient self-reporting.

\section{Concomitant treatment}

Medical treatment that did not violate trial exclusion criteria was maintained for patients having a known disease other than malaria at the beginning of the trial.

\section{Management and statistical analysis of data}

All data were initially recorded by hand in clinic notebooks, then transferred to an Excel file. Proportions were compared using chi-square test or Fisher exact test. Continuous and normally distributed data were compared by analysis of variance (ANOVA) and Student's ttest. Other non-normally distributed data were compared by Mann-Whitney $U$ test. Survival analyses, fever clearance time, and the parasite clearance time used the Kaplan-Meier method with two-sided log-rank statistics. No significant differences were seen among the five sites, so the data were pooled. Data were analyzed using SPSS for Windows version 1.0.0.903 and $\mathrm{R}$ for Windows version 3.4.2. Pvalues $<0.05$ were considered statistically significant.

\section{Results and discussion}

This trial was conducted as a superiority trial. Where equivalence was noted, it was so stated. The primary outcome of the trial was that, based on microscopic analyses, both Artemisia sp. cured malaria faster and more effectively than ASAQ. The secondary outcomes were that compared to ASAQ, Artemisia-treated patients also had microscopically undetectable gametocytes and fewer adverse side effects.

\section{Clinical admission characteristics:}

There were 943 patients who completed the trial with 258 pediatrics in each of the ASAQ and $A$. annua arms (Table 1). The median age was $29 \mathrm{y}$ in the ASAQ group, $19 \mathrm{y}$ in the $A$. annua group and $25 \mathrm{y}$ in the $A$. afra group (Table 2); ages within each group ranged from 6 to $50 \mathrm{y}$. Of these, $67.7 \%$ were male, $32.3 \%$ were female; $41.1 \%$ were pediatrics and $58.9 \%$ adults in the $A$. annua group. In the $A$. afra group, $70.9 \%$ were male, $29.1 \%$ were female; $22.9 \%$ were pediatrics and $77.1 \%$ adults. In the ASAQ group, $65.3 \%$ were male, $34.7 \%$ were female; $22.3 \%$ were pediatrics and $77.7 \%$ adults. Of the five study sites, three included all three treatment arms, one had $A$. annua and ASAQ; the other had $A$. afra and ASAQ (Table 2). At admission parasitemia was $>33,000$ trophozoites/ $\mu l$ for all treatment arms.

\section{Fever and parasitemia progression:}

Cures rates were established by D28 parasitemia. Compared to ASAQ-treated patients with an overall $34.3 \%$ cure by D28, $A$. afra and $A$. annua-treated patients were 88.8 and $96.4 \%$ cured, respectively (Table 3). Cures for ASAQ-treated patients were $49.5 \%$ for pediatrics (ages 5-15 y) and 30.0\% for adults (ages 16-65) (Table 3). Pediatric cures for $A$. annua and $A$. afra-treated patients were 91.2 and $82.4 \%$, respectively, while for adults, cures were 100 and $90.7 \%$, respectively (Table 3). There were 344 patients with parasites at D28; 9 for $A$. annua, 25 for $A$. afra, and 310 for ASAQ. Due to degradation of stored blood samples, we 
were unable to conduct a valid PCR analysis of the D28 patients with parasitemia. Considering the large number of D28 patients with parasitemia, a thorough PCR analysis is needed in future studies.

Fever clearance took $24 \mathrm{~h}$ in the Artemisia arm and $48 \mathrm{~h}$ for ASAQ (Fig. 2). Zime-Diawara et al. (2015) noticed total apyrexia even faster, only $12 \mathrm{~h}$ post-first Artemisia infusion. Using the log-rank test, $p$ values were all significantly different among all four measured groups: both Artemisias vs. ASAQ; A. annua vs. A. afra; A. апnua vs. ASAQ; $A$. afra vs.ASAQ.

On D1, parasitemia decreased $97.7 \%$ in the Artemisia arm and 85.2\% for ASAQ. In both Artemisia arms, parasitemia totally cleared on D2 (Fig. 3). In the ASAQ arm, however, parasites remained in some patients until D14. By the log-rank test at D28, three of the four compared groups were significantly different, but there was no significant difference between $A$. annua and $A$. afra $(p=0.505)$. These results are similar to Zime-Diawara et al. (2015) with 108 patients receiving $1 \mathrm{l} / \mathrm{d}$ of $12 \mathrm{~g} / \mathrm{l} A$. annua infusion. Parasitemia disappeared in all patients after $36 \mathrm{~h}$, with excellent clinical tolerance. In a similar 48-patient study, Mueller et al. (2004) observed trophozoite disappearance by D4 for $92 \%$ of patients. Our Artemisia-treated patients were $96.4 \%$ cured on D28; only 5\% of patients showed side effects, which was significantly better than ASAQ-treated patients with $42.8 \%$ adverse side effects (Table 4).

Patients treated with ASAQs received weight-adjusted amounts of artesunate ranging from 147.2 to $1293 \mathrm{mg}$ in toto. Patients treated with $A$. afra received a maximum amount of artemisinin of $1.26 \mathrm{mg}$, while those treated with $A$. annua received $46.9 \mathrm{mg}$ (BUR) or 59.5 $\mathrm{mg}$ (LUX) in toto; there was no weight adjustment. This was 3-1000 fold less artemisinin vs. ASAQs, yet Artemisia therapeutic outcome was better. This was consistent with recent case studies (Daddy et al., 2017) demonstrating efficacy of $A$. annua compressed leaf tablets (DLA) in treating patients unresponsive to either ASAQ or i.V. artesunate. The treatment course of DLA used in that study provided $55 \mathrm{mg}$ maximum artemisinin to adults; for pediatrics, it was as low as $13.75 \mathrm{mg}$. These results collectively suggest other phytochemicals in the plants were crucial to the potent therapeutic outcome of the Artemisias.

\section{Gametocyte elimination:}

D0 gametocyte carriers present in ASAQ-treated patients declined beginning on D7 posttreatment; by D28 only 10 of 472 patients were detectable carriers (Fig. 4). In contrast, no gametocyte carriers were detected in either Artemisia treatment from D14 onwards suggesting that both Artemisias, but not ASAQ, eliminated gametocytes. Gametocyte carriage was completely eliminated in all age groups treated with Artemisia (Table 5). In ASAQ-treated patients, both pediatric and adult patients still had microscopically detectable gametocytes at D28 (Table 5). Microscopic analysis underestimates gametocyte counts by several fold (Babiker and Schneider 2008), so future studies should use nested PCR to further probe for the presence of gametocytes in Artemisia-treated patients.

Gender differences occurred in the ASAQ group. There were 326 males in the Artemisia arm and 308 in the ASAQ arm, and 145 females in the Artemisia arm and 164 in the ASAQ 
arm. While both genders responded equally well to Artemisia, there was significantly more gametocyte carriage in females than males in the ASAQ-treated arm for D14-28 (Fisher's Exact test, $p=0.012$; data not shown). There was no differential age response in females. Artemisia-treated male carriers were significantly different at D2-4 but not at D7-28. Overall, these results indicated that Artemisias were better than ASAQ at removing gametocytes in females.

\section{Hemoglobin improvement by A. afra, but not ASAQ or A. annua:}

While hemoglobin at D0, 1, 3, and 4 between $A$. annua and ASAQ was not statistically different (post hoc test, $p>0.05$ ), between $A$. annua and $A$. afra there was a significant difference (Fig. 5). Hemoglobin remained significantly greater at about $11 \mathrm{~g} / \mathrm{dl}$ post treatment for $A$. afra $v s . \sim 9$ and $9.5 \mathrm{~g} / \mathrm{dl}$ for ASAQ and $A$. annua, respectively. This similarity between ASAQ and $A$. annua is likely the result of artemisinin, known to reduce hemoglobin during treatment (Kurth et al., 2016). A. afra has but a trace of artemisinin (see Table 1 in Munyangi et al. (2018) for artemisinin content of each Artemisia sp. treatment) and thus, as expected, there was no decline in hemoglobin.

\section{ALAT and ASAT were not altered:}

Although liver transaminases are often altered in malaria patients (Woodford et al., 2018), there were no significant differences observed in ALAT and ASAT among the three treatment arms (see supplemental Figure S1).

\section{Other studies using Artemisia tea infusions:}

In China the traditional treatment against malaria is a daily dose from 4.5 to $9 \mathrm{~g}$ of dry leaves and twigs of $A$. annua infused in boiling water. In 1972, the Institute for Traditional Medicine of Beijing treated 31 patients with an ether extract; 20 had $P$. vivax infection, 9 had $P$. falciparum, and 2 patients had both. Fever cleared in $36 \mathrm{~h}$ for the $P$. falciparum-infected patients (Zhang 2005; Cui and Su 2009). In another instance, Chang and But (1986) administered $72 \mathrm{~g}$ of $A$. annua in an alcoholic extract over $3 \mathrm{~d}$ on 485 patients infected with $P$. falciparum, and on 105 patients with $P$. vivax. The cure rate was $100 \%$ against both malaria species. In 2000, Mueller et al. (2000) treated 53 DRC patients infected with $P$. falciparum using two different treatments: infusion and decoction. The posology was $5 \mathrm{~g}$ of dried leaves in 11 over $5 \mathrm{~d}$ The average cure rate was $92 \%$ after $4 \mathrm{~d}$ of treatment. In Ethiopia, Gebeyaw et al. (2010) treated 73 patients suffering from uncomplicated malaria with 11 of $A$. annua dried leaf infusion using $5 \mathrm{~g} / 1$ per d over $7 \mathrm{~d}$; $95.5 \%$ of patients were cured without adverse effects. Chougouo-Kengne et al. (2012) compared $A$. annua infusion with artesunate alone or combined with amodiaquine on 73 patients suffering from uncomplicated falciparum malaria. The posology was $5 \mathrm{~g}$ of dried leaf infusion in boiling water, over 5 or $7 \mathrm{~d}$. The $7 \mathrm{~d}$ treatment improved the results from $71.3 \%$ to $100 \%$, without any side effects. Overall, $7 \mathrm{~d}$ of treatment with $5 \mathrm{~g} / \mathrm{l}$ Artemisia annua infusion seemed to provide the best therapeutic outcome. 


\section{Synergistic effects:}

In prior tea infusion studies, there was efficacy of the $A$. annua infusion against uncomplicated malaria without significant side effects. This is in line with our results showing a cure rate of $99.5 \%$ on D28 for Artemisia with only 5\% side effects. Previously, artemisinin was considered the only active molecule, so prior studies tried to reach high levels in their preparations. Artemisinin is only present in traces in some Artemisia varieties and usually absent in $A$. afra. This of course raises the question of the role of artemisinin in these infusions used to treat malaria. $A$. annua and $A$. afra contain hundreds of phytochemical molecules and minerals. Antimalarial activity has been demonstrated for some 20 of these as summarized in the review by Weathers et al. (2014). It is important to note that $A$. afra has only trace amounts of artemisinin and no detectable artemisinic parent molecules (Munyangi et al., 2018), yet almost all patients treated with that species had the same therapeutic response as those treated with $A$. annua.

\section{Artemisinin drug resistance:}

Despite the fact that $A$. annua shows high efficacy without side effects, there has been reluctance in endorsing use of the plant. Low artemisinin content of the plant elicited concerns that this might induce artemisinin drug resistance (Blanke et al., 2008; Mueller et al., 2004; WHO, 2012 http://www.who.int/malaria/ position_statement_herbal_remedy_artemisia_annua_l.pdf). Since then rodent studies using per os dried leaf Artemisia (DLA), Elfawal et al. (2015) demonstrated that compared to pure artemisinin, DLA was threefold more resilient against evolution of artemisinin drug resistance. Furthermore, DLA even eliminated artemisinin-resistant parasites in a $P$. yoelli rodent model (Elfawal et al., 2015). In humans unresponsive to either ASAQ or i.v. artesunate, $A$. annua (DLA) eliminated parasites within a few days even in comatose pediatrics (Daddy et al., 2017). Studies showed that the numerous phytochemicals in $A$. annua and $A$. afra provide synergies and multiple interactions, likely constituting a polytherapy against malaria (Elford et al., 1987; Li et al., 2018; Liu et al., 1992; Suberu et al., 2013). Together, those data and this study indicate that artemisinin is not the sole phytochemical demonstrating antimalarial efficacy by Artemisia sp. Moreover, the presence of multiple phytochemicals in Artemisia sp. acts not as a mono or dual therapy, but as a poly-combination-therapy.

In the present study, we saw a large number of ASAQ failures, yet in the same localities, $A$. afra and especially $A$. annua were highly effective. If those failures are ASAQ resistance, then Artemisia tea infusions cured those patients. As we and others observe mounting failures of ACTs in Africa, Artemisia annua in particular could provide an effective and rapid alternative for holding the parasite at bay. For how long? No one knows, but lives are at risk and this is an inexpensive, safe and effective therapeutic that could be implemented rather quickly.

\section{Conclusion}

Treating uncomplicated malaria with either $A$. annua or $A$. afra was superior to the artesunate-amodiaquine ASAQ treatment. Fever and parasitemia clearances were faster and 
more efficient with both Artemisia species than with ASAQ; adverse effects were negligible. At D14-28 gametocyte carriage was undetectable in Artemisia-treated patients, so transmission to the mosquito should be interrupted. Artemisia is a polytherapy with at least 10 active molecules likely acting in synergy, so resistance is therefore unlikely to emerge. Plants can be grown and used by local populations, which could help counteract the problems caused by fake or obsolete antimalarial drugs. Despite these exciting results, however, it is important that further large-scale studies be launched to optimize use of these plants and posology for children, pregnant women and others, in the case of uncomplicated as well as severe malaria.

\section{Supplementary Material}

Refer to Web version on PubMed Central for supplementary material.

\section{Acknowledgments}

Authors appreciate Impala Avenir Foundation, Mme Hélène de Cossé Brissac and Mr. Jean-Louis Bouchard for generously funding this study. Matthew Desrosiers, Danielle Snider, and Dr. Bruno Giroux provided valuable feedback. We are also grateful for Award Number 2NIH-R15AT008277-02 from the National Center for Complementary and Integrative Health that enabled PJW and MJT to provide phytochemical analyses of the plant material used in treating these patients. The content is solely the responsibility of the authors and does not necessarily represent the official views of the National Center for Complementary and Integrative Health or the National Institutes of Health.

\section{Abbreviations:}

ACT

ASAQ

DLA

i.v.

PNLP artemisinin combination therapy

artesunate-amodiaquine

dried leaf Artemisia

intravenous

programme national de Lutte contre le Paludisme

\section{References}

Blanke CH, Naisabha GB, Balemea MB, Mbaruku GM, Heide L, Müller MS, 2008 Herba Artemisiae annuae tea preparation compared to sulfadoxinepyrimethamine in the treatment of uncomplicated falciparum malaria in adults: a randomized double-blind clinical trial. Trop. Doct 38, 113-116. 10.1258/td.2007.060184. [PubMed: 18453510]

Babiker HA, Schneider P, 2008 Application of molecular methods for monitoring transmission stages of malaria parasites. Biomed. Mater 3 (3), 034007. [PubMed: 18708712]

Chang HM, But PPH, 1986 Pharmacology and Applications of Chinese Materia Media Volume 1 World Scientific Publishing, Singapore.

Chougouo-Kengne RD, Kouamouo J, Mouyou-Somo R, Penge On' Okoko A, 2012 Etude comparative de l'efficacité thérapeutique de l'Artesunate seul ou en association avec l'amodiaquine et de la tisane de Artemisia annua cultive à l'ouest du Cameroun. Annales de Pharmacie de l'Université de Kinshasa (RDC) 4 (1), 129-150.

Cui L, Su XZ, 2009 Discovery, mechanisms of action and combination therapy of Artemisinin, Expert. Rev. Anti. Infect. Ther. Oct 7 (8), 999-1013. 10.1586/eri.09.68. 
Daddy BN, Kalisya ML, Bagire GP, Watt RL, Towler MJ, Weathers PJ, 2017 Artemisia annua dried leaf tablets treated malaria resistant to ACT and i.v. artesunate: Case reports. Phytomedicine 32, 3740. 10.1016/j.phymed.2017.04.006. [PubMed: 28732806]

Elfawal MA, Towler MJ, Reich NG, Weathers PJ, Rich SM, 2015 Dried whole plant Artemisia annua slows evolution of malaria drug resistance and overcomes resistance to artemisinin. PNAS USA 112, 821-826. 10.1073/pnas.1413127112. [PubMed: 25561559]

Elford BC, Roberts MF, Phillipson D, Wilson RJM, 1987 Potentiation of the antimalarial activity of qinghaosu by methoxylated flavones. Trans. R. Soc. Trop. Med. Hyg 81, 434-436. [PubMed: 3318019]

Gathirwa JW, Rukunga GM, Njagi ENM, Omar SA, Guantai AN, Muthaura CN, Mwitari PG, Kimani CW, Kirira PG, Tolo FM, Ndunda TN, Ndiege IO, 2007 In vitro anti-plasmodial and in vivo antimalarial activity of some plants traditionally used for the treatment of malaria by the Meru community in Kenya. J. Nat. Med 61, 261-268. 10.1007/s11418-007-0140-0.

Gebeyaw T, Kebede Y, Yigzaw T, 2010 Use of the plant Artemisia annua as a natural anti-malarial herb in Arbaminch town. Ethiop. J. Health Biomed. Sci 2, 75-82.

Kraft C, Jenett-Siems K, Siems K, 2003 In vitro antiplasmodial evaluation of medicinal plants from Zimbabwe. Phytother. Res 70, 123-128.

Kurth F, Lingscheid T, Steiner F, Stegemann MS, Bélard S, Menner N, Pongratz P, Kim J, von Bernuth H, Mayer B, Damm G, Seehofer D, Salama A, Suttorp N, Zoller T, 2016 Hemolysis after oral artemisinin combination therapy for uncomplicated Plasmodium falciparum malaria. Emerg. Infect. Dis 22, 1381-1386. 10.1016/j.ijid.2014.09.007. [PubMed: 27434054]

Liu KC, Yang SL, Roberts MF, Elford BC, Phillipson JD, 1992 Antimalarial activity of Artemisia annua flavonoids from whole plants and cell cultures. Plant Cell Rep. 11, 637-640. 10.1007/ BF00236389. [PubMed: 24213368]

Li J, Zhang C, Gong M, Wang M, 2018 Combination of artemisinin-based natural compounds from Artemisia annua L. for the treatment of malaria: Pharmacodynamic and pharmacokinetic studies. Phytother. Res 1-6 2018.

Liu NQ, Cao M, Frédérich M, Choi YH, Verpoorte R, Van der Kooy F, 2010 Metabolomic investigation of the ethnopharmacological use of Artemisia afra with NMR spectroscopy and multivariate data analysis. J. Ethnopharmacol 128, 230-235. 10.1016/j.jep.2010.01.020. [PubMed: 20079415]

Moyo P, Botha ME, Nondaba S, Niemand J, Maharaj VJ, Eloff JN, Louw AI, Birkholtz L, 2016 In vitro inhibition of Plasmodium falciparum early and late stage gametocyte viability by extracts from eight traditionally used South African plant species. J. Ethnopharmacol 185, 235-242. [PubMed: 26994818]

Mueller MS, Karhagomba IB, Hirt HM, Wemakor E, 2000 The potential of Artemisia annua L. as a locally produced remedy for malaria in the tropics: Agricultural, chemical and clinical aspects. J. Ethnopharmacol 73, 487-493. 10.1016/j.jep.2016.03.036. [PubMed: 11091003]

Mueller MS, Runyambo N, Wagner I, Borrmann S, Dietz K, Heide L, 2004 Randomized controlled trial of a traditional preparation of Artemisia annua L. (Annual Wormwood) in the treatment of malaria. Trans. R. Soc. Trop. Med. Hyg 98, 318-321. [PubMed: 15109558]

Munyangi J, Cornet-Vernet L, Idumbo M, Lu C, Lutgen P, Perronne C, Ngombe N, Bianga J, Mupenda B, Lalukala P, Mergeai G, Mumba D, Towler M, Weathers P, 2018 Effect of Artemisia annua and Artemisia afra tea infusions on schistosomiasis in a large clinical trial. Phytomedicine 51, 233 240. 10.1016/j.phymed.2018.10.014. [PubMed: 30466622]

Smith LMJ, Bentley S, Jones H, Burns C, Aroo RRJ, Woolley JG, 2010 Developing an alternative UK industrial crop Artemisia annua, for the extraction of artemisinin to treat multi-drug resistant malaria. Aspects Appl. Biol 101, 99-106.

Suberu JO, Gorka AP, Jacobs L, Roepe PD, Sullivan N, Barker GC, Lapkin AA, 2013 Anti-plasmodial polyvalent interactions in Artemisia annua L. aqueous extract-possible synergistic and resistance mechanisms. PLoS One 8, e80790 10.1371/journal.pone.0080790. [PubMed: 24244716]

Weathers PJ, Reed K, Hassanali A, Lutgen P, Engeu PO, 2014 Chapter 4: Whole plant approaches to therapeutic use of Artemisia annua L. (Asteraceae) In: Aftab T, Ferreira JFS, Khan MMA, Naeem 
(Eds.), Artemisia annua. - Pharmacology and Biotechnology. Springer, Heidelberg, GDR, pp. 5174.

Willcox M, Bodeker G, Bourdy G, Dhingra V, Falquet J, Ferreira JFS, Graz B, Hirt H-M, Hsu E, de Magalhães PM, Provendier D, Wright CW, 2004 Artemisia annua as a traditional herbal antimalarial In: Willcox ML, Bodeker G, Rasoanaivo P (Eds.), Traditional Medicinal Plants and Malaria. CRC Press, Boca Raton, FL, pp. 43-59.

Woodford J, Shanks GD, Griffin P, Chalon S, McCarthy JS, 2018 The dynamics of liver function test abnormalities after malaria infection: A retrospective observational study. Am. J. Trop. Med. Hyg 98, 1113-1119. [PubMed: 29436349]

Zhang JF, 2005 A Detailed Chronological Record of Project 523 and the Discovery and Development of Qinghaosu (Artemisinin) Vol. 193 Yangcheng Evening News Publishing Company, China.

Zime-Diawara H, Sissinto-Savi de Tove Y, Okogbeto OE, Ogouyemi-Hounto A, Gbaguidi FA, KindeGazard D, Massougbodji A, Bigot A, Sinsin B, Quetin-Leclerc J, Evrard B, Moudachirou M, 2015 Etude de l'efficacité et de la tolérance d'une tisane à base d'Artemisia annua L. (Asteraceae) cultivée au Bénin pour la prise en charge du paludisme simple. Int. J. Biol. Chem. Sci 9 (2), 692 702. 


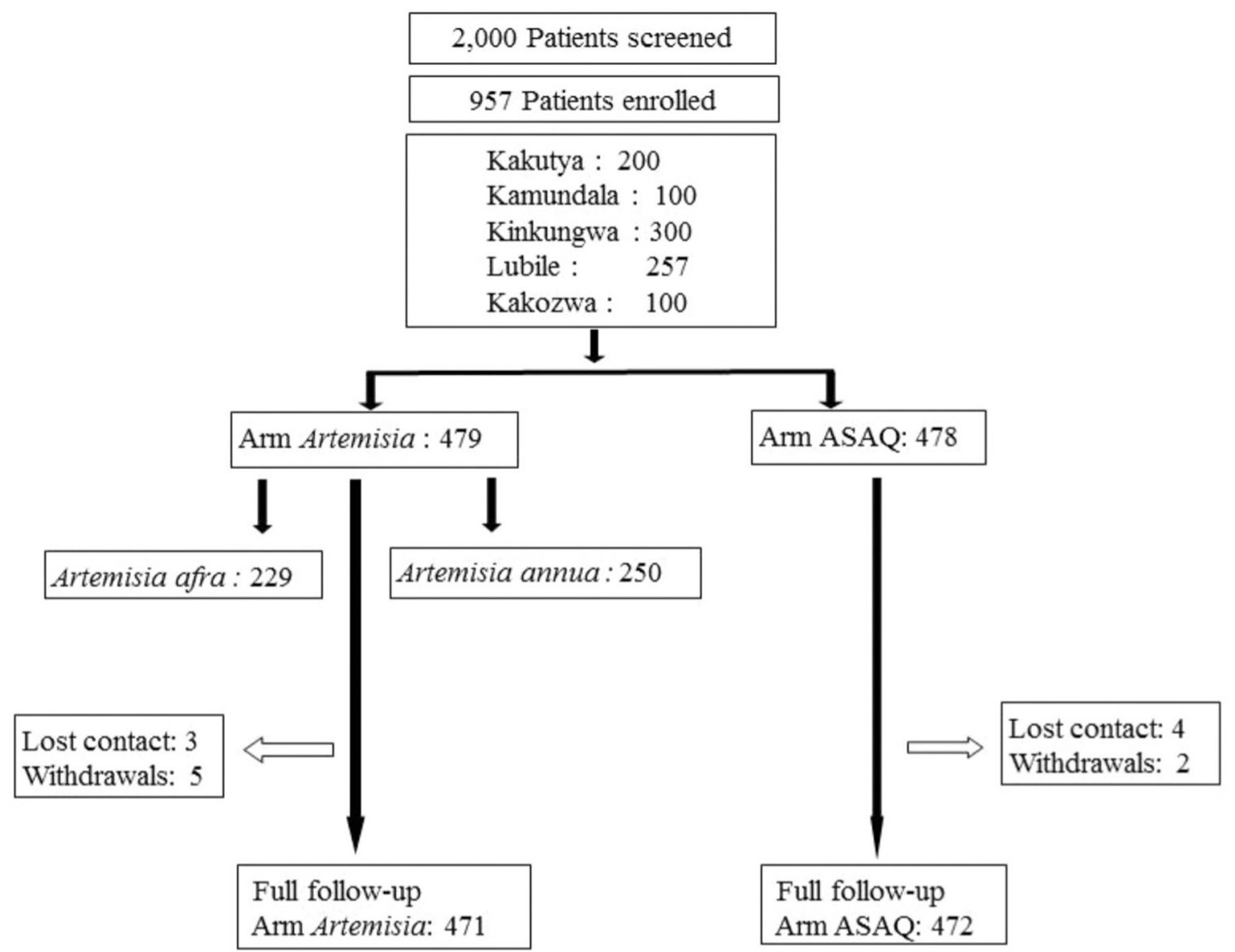

Fig. 1.

Trial design. 

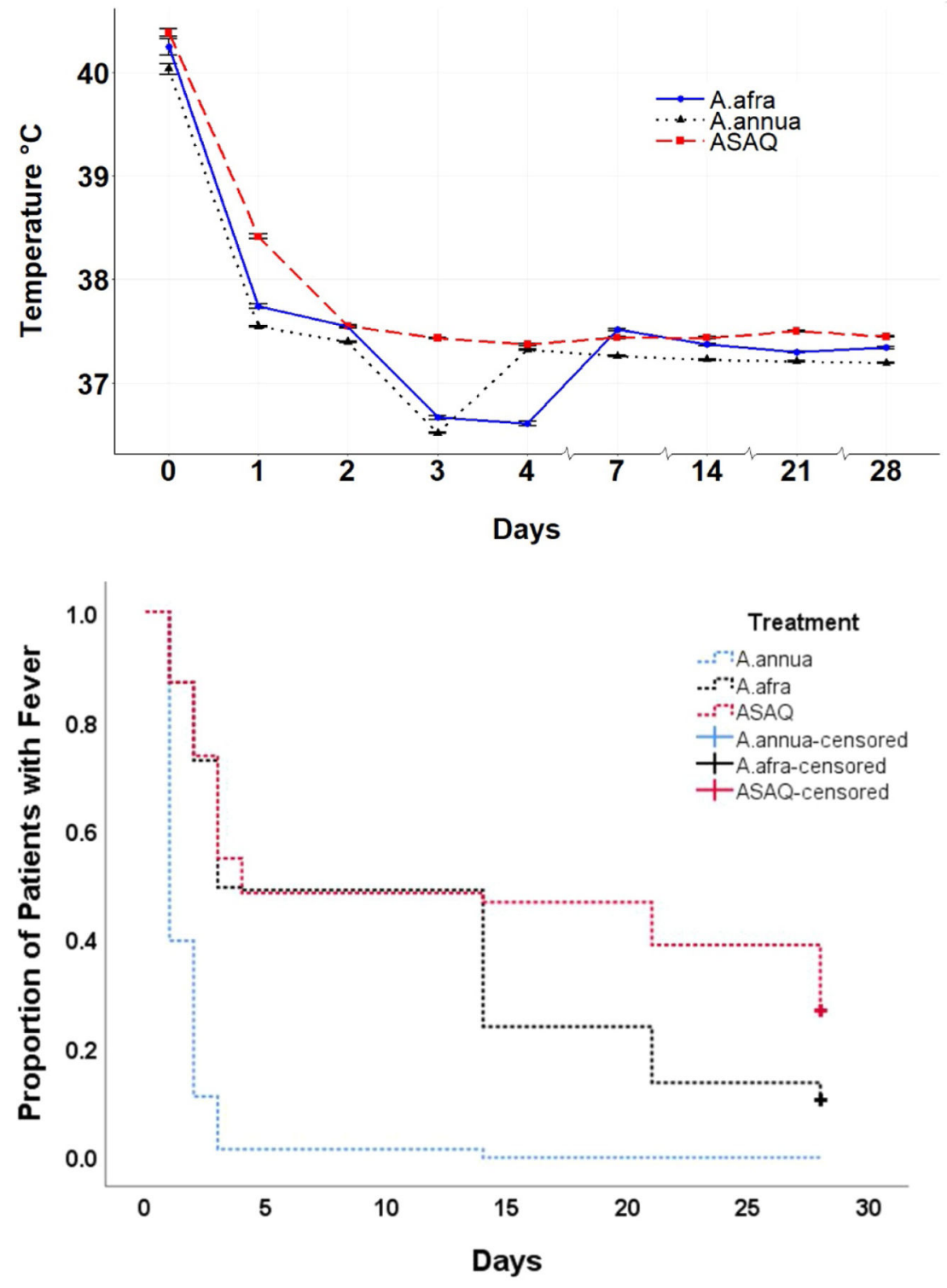

Fig. 2.

Average fever progression among the three treatment arms. Top, graphical representation. Bottom, Kaplan-Meier; survival time of fever started when a patient was included in the study (D0). Patients were followed until D28. For patients withdrawing from the study before D28 or still having fever until D28, survival time is said to be censored. The survival probability was calculated using the Kaplan-Meier method. The censored rates of $A$. annua, $A$. afra, and ASAQ are $0.0 \%, 10.3 \%$, and $26.7 \%$, respectively, with $p$-value of log-rank still near to zero and statistically significant. 

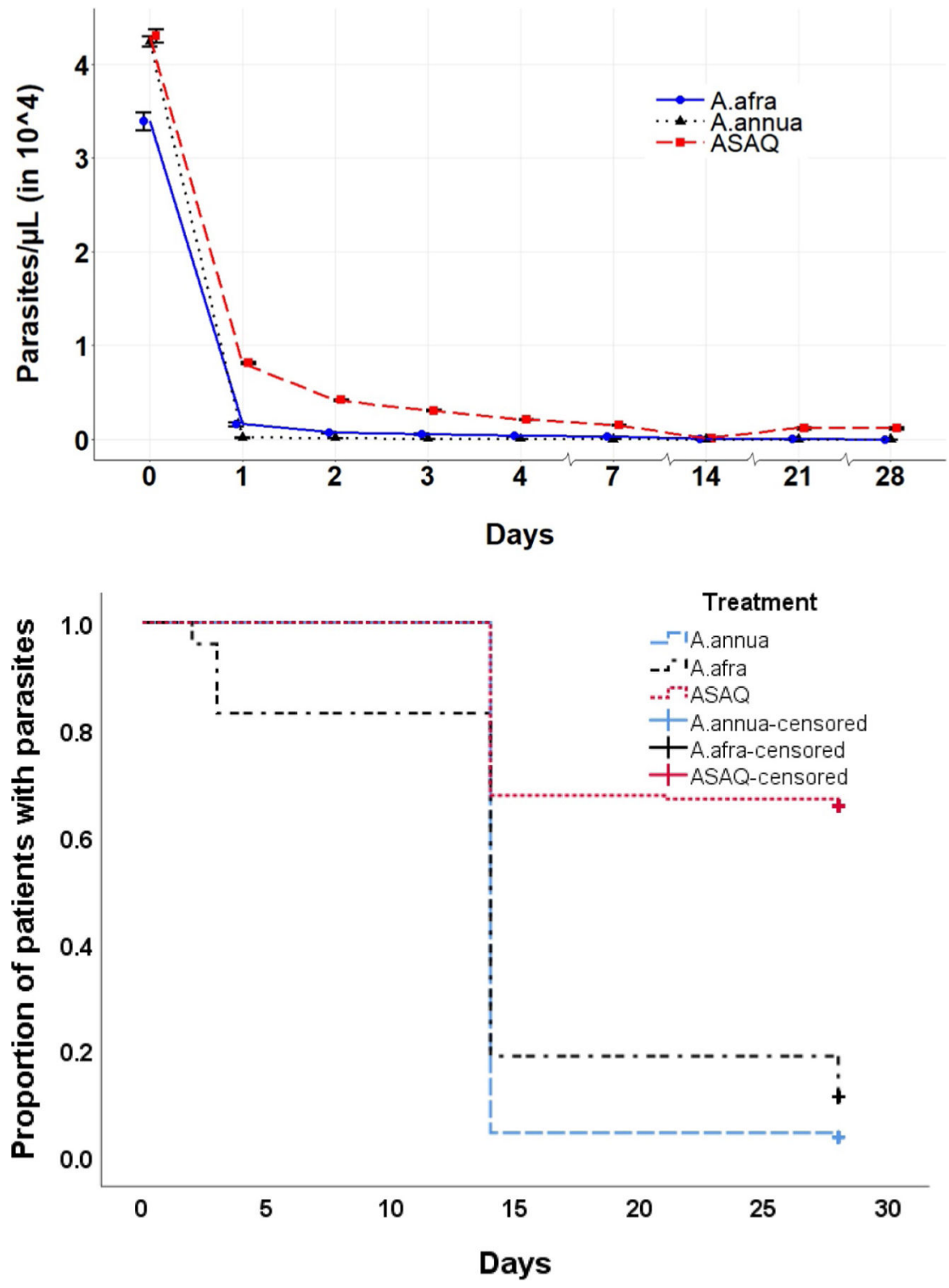

Fig. 3.

Average parasitemia progression among the three treatment arms. Top, graphical representation. Bottom, Kaplan-Meier; survival time of parasites started when a patient was included in the study (D0). Patients were followed until D28. For patients withdrawing from the study before D28 or still having parasites until D28, survival time is said to be censored. The censored survival rates of $A$. annua, $A$. afra, and ASAQ are $3.6 \%, 11.2 \%$, and $65.7 \%$, respectively. 


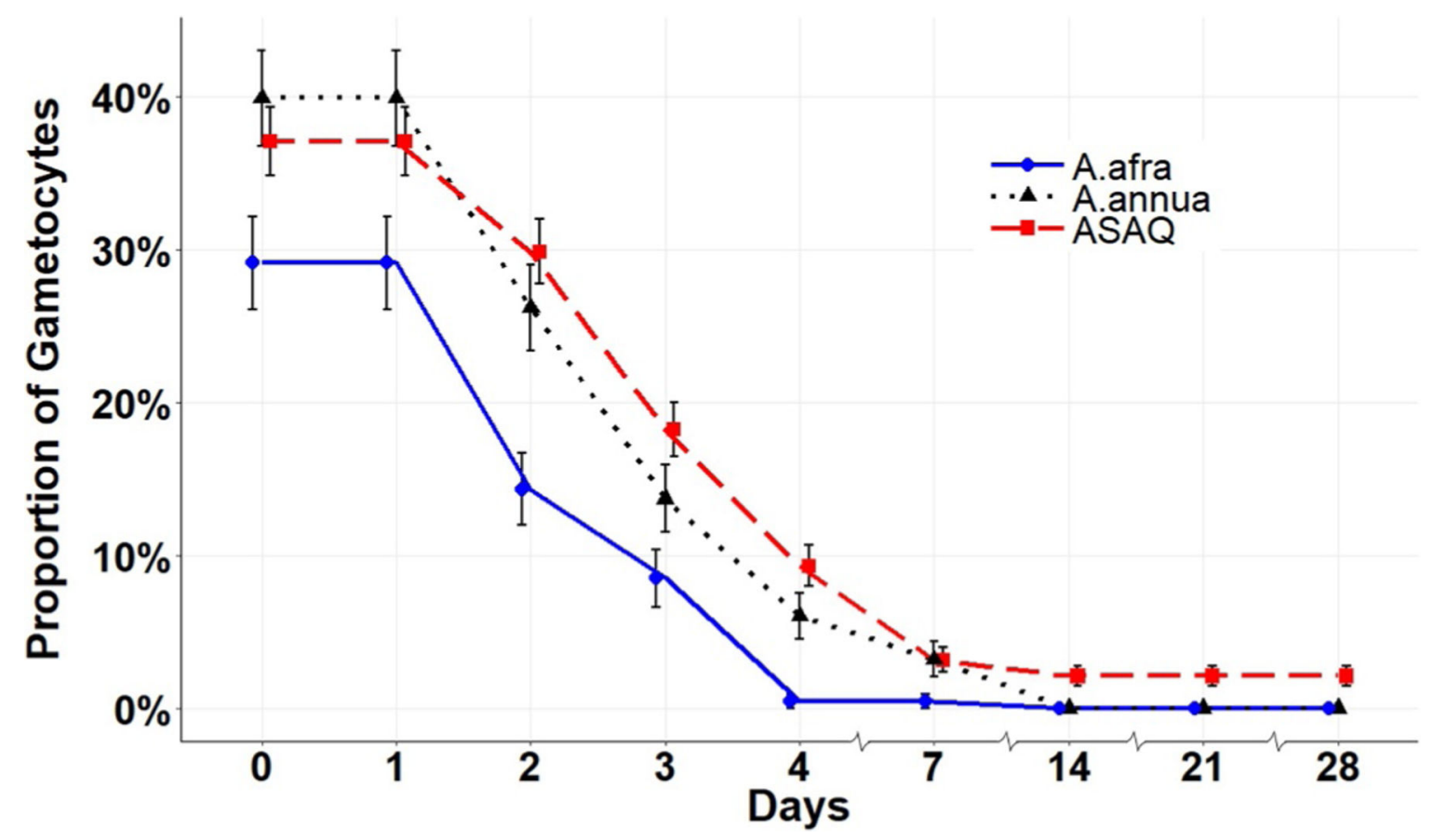

Fig. 4.

Microscopically determined proportion of patients with gametocytes (carriers) throughout the trial period. 


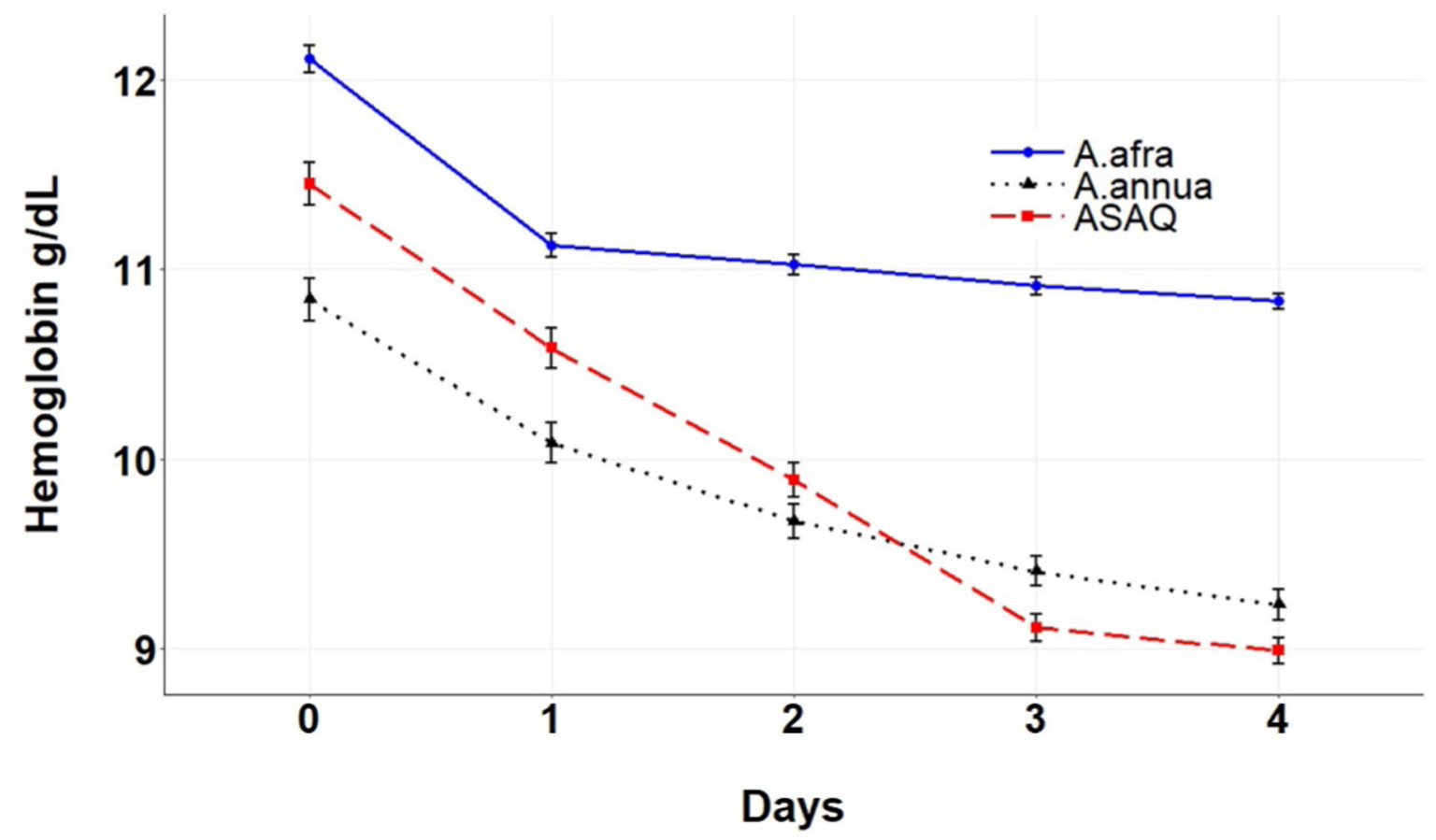

Fig. 5.

Hemoglobin levels during the first four days of treatment. 

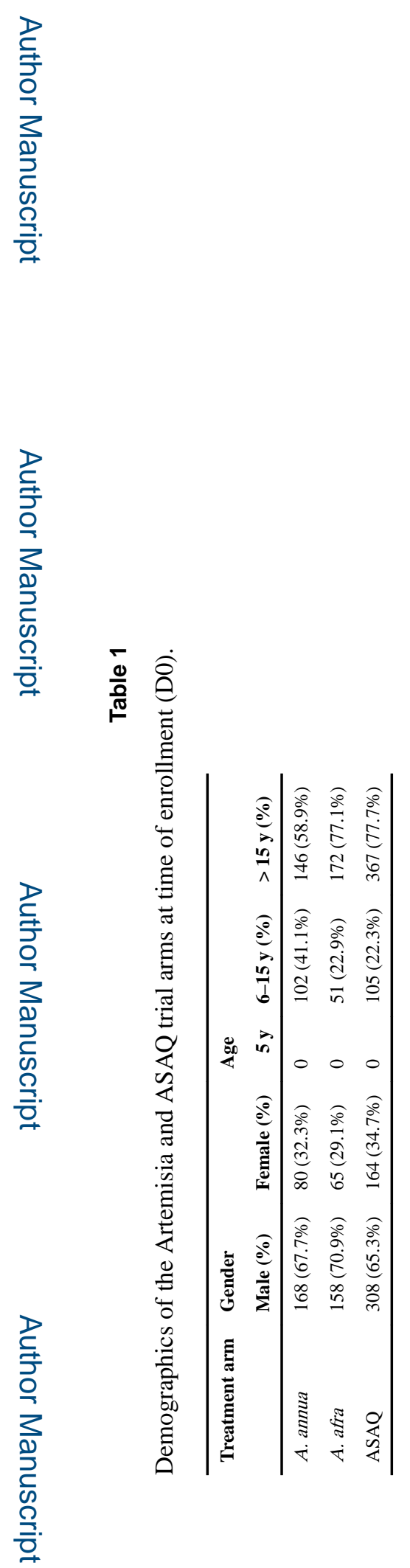

Phytomedicine. Author manuscript; available in PMC 2020 April 01. 
Table 3

Cure rates by age group within each trial arm at D14 and 28 .

\begin{tabular}{|c|c|c|c|c|c|c|}
\hline \multirow[t]{2}{*}{ Age (y) } & \multicolumn{3}{|l|}{ D14 } & \multicolumn{3}{|l|}{ D28 } \\
\hline & A. апnиа $\mathrm{n} / \mathrm{N}^{a}(\%)^{b}$ & A. afra $\mathbf{n} / \mathbf{N}(\%)$ & ASAQ n/N (\%) & A. апnиа $\mathrm{n} / \mathrm{N}(\%)$ & $A$. afra $\mathrm{n} / \mathrm{N}(\%)$ & ASAQ n/N (\%) \\
\hline \multirow[t]{2}{*}{$5-15$} & $93 / 102$ & $36 / 51$ & $51 / 105$ & $93 / 102$ & $42 / 51$ & $52 / 105$ \\
\hline & $(91.2 \%)$ & $(70.6 \%)$ & $(48.6 \%)$ & $(91.2 \%)$ & $(82.4 \%)$ & $(49.5 \%)$ \\
\hline \multirow[t]{2}{*}{$16-65$} & $146 / 146$ & $145 / 172$ & $110 / 367$ & $146 / 146$ & $156 / 172$ & $110 / 367$ \\
\hline & $(100.0 \%)$ & $(84.3 \%)$ & $(30.0 \%)$ & $(100.0 \%)$ & $(90.7 \%)$ & $(30.0 \%)$ \\
\hline \multirow[t]{2}{*}{ Overall } & $239 / 248$ & $181 / 223$ & $161 / 472$ & $239 / 248$ & $198 / 223$ & $162 / 472$ \\
\hline & $(96.4 \%)$ & $(81.2 \%)$ & $(34.1 \%)$ & $(96.4 \%)$ & $(88.8 \%)$ & $(34.3 \%)$ \\
\hline
\end{tabular}

${ }^{a} \mathrm{~N}$, total number within age group less any who left the trial; n, number with 0 parasitemia.

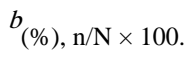


Table 4

Distribution among patients of adverse effects from treatment.

\begin{tabular}{lll}
\hline Observed adverse effects & Number of subjects in the Artemisia arms & Number of subjects in the ASAQ arm \\
\hline Abdominal pain & 0 & 25 \\
Asthenia & 0 & 30 \\
Diarrhea & 0 & 5 \\
Drowsiness & 0 & 3 \\
Fatty cough & 0 & 1 \\
Hypoglycemia & 0 & 20 \\
Insomnia & 0 & 10 \\
Nausea & 10 & 30 \\
Pruritis & 0 & 35 \\
Vertigo & 0 & 1 \\
Vomiting & 15 & 50 \\
Total & 25 & 210 \\
$\%$ of total & $5.0 \%$ & $42.8 \%$ \\
\hline
\end{tabular}

Phytomedicine. Author manuscript; available in PMC 2020 April 01. 
Table 5

Level of microscopically determined gametocyte carriage decrease D14-28 within age groups.

\begin{tabular}{llll}
\hline Age (y) & A. annua $\mathbf{n} / \mathbf{N}(\%)$ & A. afra $\mathbf{n} / \mathbf{N}(\%)$ & ASAQ n/N (\%) \\
\hline Children (5-15) & $43 / 43(100 \%)$ & $102 / 102(100 \%)$ & $111 / 114(97.4 \%)$ \\
Adults (16-65) & $205 / 205(100 \%)$ & $147 / 147(100 \%)$ & $369 / 376(98.1 \%)$ \\
Total & $248 / 248(100 \%)$ & $249 / 249(100 \%)$ & $480 / 490(98.0 \%)$ \\
\hline
\end{tabular}

$\mathrm{N}$, total within age group; $\mathrm{n}$, number of patients with microscopically undetectable gametocytes. 